\title{
Pharmacist as Pharmacovigilance Practitioner
}

\author{
Vijay Venkatraman Janarthanan*, Ganesan Ramakrishnan, Subashchander Krishnamurthy and \\ Akash Imayam Sahar
}

Oviya MedSafe Pvt. Ltd, $2^{\text {nd }}$ Floor, KTVR Gardens 220a-3, Marudha Konar Road Velandipalayam, Coimbatore-641 025, Tamil Nadu, INDIA.

\begin{abstract}
Pharmacy as a profession has made significant progress in the recent years. It has seen a paradigm shift from product orientation to patient focus. Pharmacist should realize it's no more a task carried out behind the curtains. This article introduces aspiring pharmacists to one such profession where they can extend their roles as providers of service which promotes drug safety.
\end{abstract}

Key words: ADR, Drug safety, Pharmacovigilance, Pharmacist, PVPI.

\section{ERSTWHILE AND TODAY}

Pharmacy is not mere preparing or dispensing of drugs. The professional practice reaches far beyond serving community. Since the inception of the concept "pharmaceutical care," coined by C. D. Hepler and L. M. Strand, the profession has seen itself extending far beyond dispensing. It emphasizes and recognizes the role of pharmacist as an integral part of the healthcare system. WHO explains pharmaceutical care as "a philosophy of practice in which the patient is the primary beneficiary of the pharmacist's actions. Pharmaceutical care focuses on the attitudes, behaviors, commitments, concerns, ethics, functions, knowledge, responsibilities and skills of the pharmacist on the provision of drug therapy with the goal of achieving definite therapeutic outcomes toward patient health and quality of life."

Speaking globally, the role of pharmacist has changed or grown, perhaps now it has become more patient-centric. Pharmacists have an important responsibility in monitoring the ongoing safety of medicines as part of their professional practice. The agreed common aspect known about pharmacists is counseling patients on potential side effects. This one-on-one two-way interaction can be utilized further by asking them to report back any undesirable occurrence which can serve as an effective feedback system. Adding to this, pharmacist's expertise can play a vital role in early detection of unintended side effects. But does that indicate drugs are unsafe? -definitely not. A drug needs to be considered as a concept, with benefit-risk profile, applied to treat patients through proven or commonly agreed measures where benefits outweigh risks. Unless otherwise proven, mere existence of negative data can never be conclusive or deemed irrelevant for further use. Numbers speak volumes about ubiquity and are never actual. A drug's benefit-risk profile is better derived from clinical judgment. Here comes the role of pharmacists on a global perspective called Pharmacovigilance.

\section{PHARMACIST AND DRUG SAFETY}

Submitted date :02-Feb-2015 Accepted date :11-Mar-2015

DOI: $10.5530 / \mathrm{ijopp} .8 .1 .2$

Address for correspondence: Dr. Vijay Venkatraman Janarthanan,

MBBS, F. Diab., MBA, FPIPA (UK), $2^{\text {nd }}$ Floor, KTVR Gardens 220a-3, Marudha Konar Road

Velandipalayam, Coimbatore $-641025$

Phone No:0422-2444442

FaxNo :0422-4203993 Tamil Nadu, INDIA.

E-mail:vijay.j@oviyamedsafe. com

History of drug goes way back to 5000 B.C with suggested use of opium. But not until 1960s after the thalidomide tragedy that it was deemed necessary to closely control the quality, efficacy and safety of medicines. This ultimately led to the discipline called pharmacovigilance.

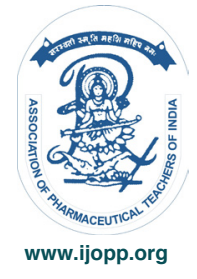




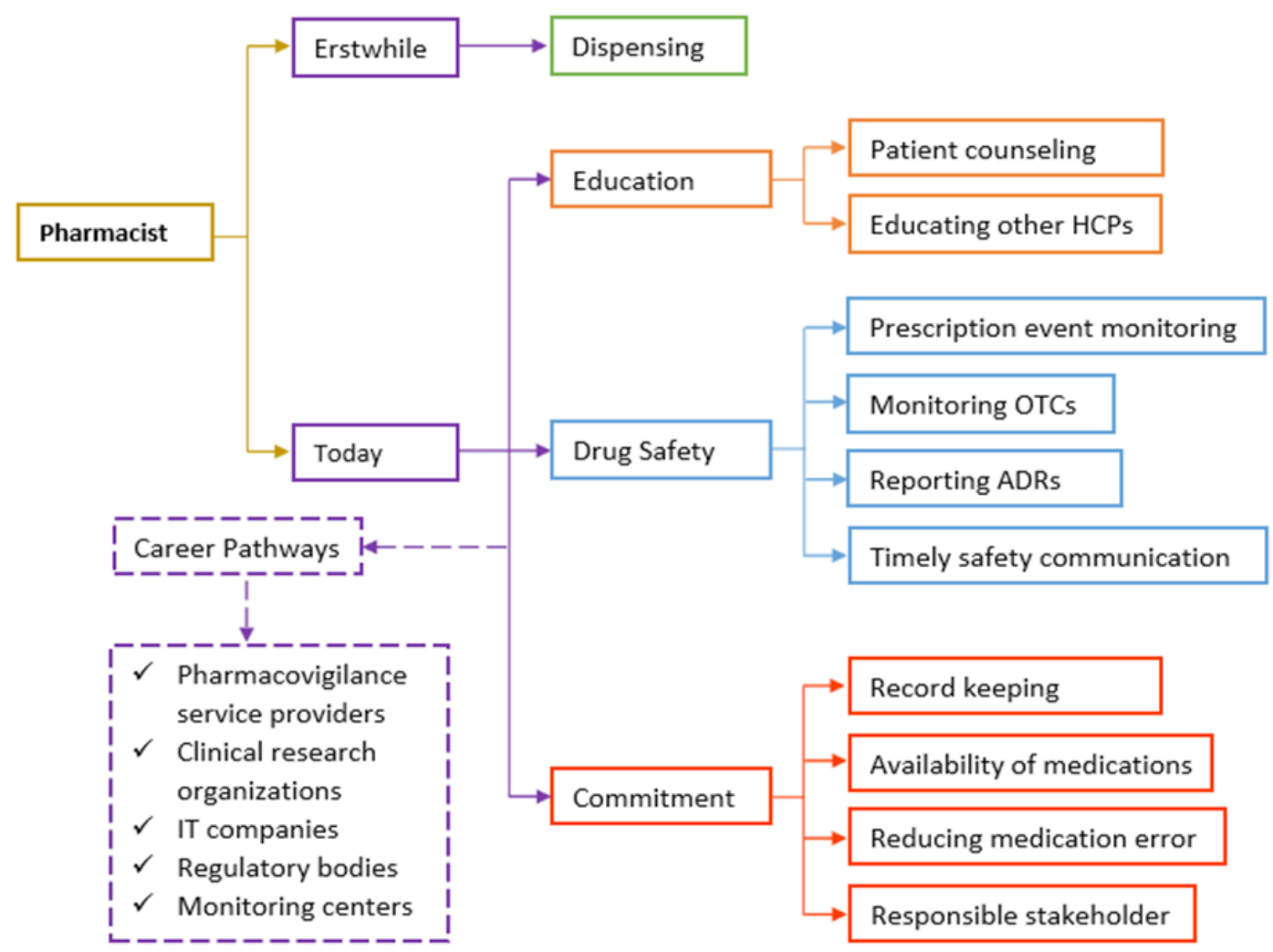

Graphical Abstract

WHO defines pharmacovigilance as science and activities relating to the detection, assessment, understanding and prevention of adverse effects or any other medicine-related problem. It's an activity contributing to the protection of patients' and public health. It would be prudent to say pharmacovigilance deals with the collection of adverse drug reaction (ADR) reports from various stakeholders responsible for monitoring the safety profile of the drug. Adverse drug reaction is a noxious and unintended response to medicinal product. It may arise from use of the product within or outside the terms of the marketing authorization, and a pharmacist knows it better why drugs are non-inclusive of safety profile in entirety before it enters market. Pharmacists have an important contribution to make with regards to postmarketing surveillance. Whether it is a non-prescription drug or a prescribed therapy, most drugs accessible to the patients are made available through the pharmacists. India still has traditional pharmacy practice where it sees more floating prescription and over-the-counter (OTC), which remains a challenge.

\section{ON THE FAR SIDE}

So, how can pharmacists be proactively involved? Well, the answer is simple. The whole purpose of pharmaco- vigilance is to minimize the potential for harm that is associated with the drug. Pharmacists form the core of interdisciplinary healthcare system where safety monitoring is the integral part of clinical practice. It would be a myth to consider a drug entirely safe and a misperception to consider it entirely harmful. From pharmacovigilance perspective, healthcare professionals need both good clinical judgment of the adverse drug reaction and sound insight into effectiveness of the drug to arrive upon the metacenter creating a relationship between benefit and risk.

\section{SAFETY AS A CONCEPT}

Safety when defined can be "relative absence of harm" but that doesn't mean safety is never doing anything and hoping nothing has happened. In pharmacovigilance, safety means collection of reports of adverse effects of drug. Safety can mean generating data and arriving upon a solution to decide further usage of drug. Apart from the routine ADRs being reported, a pharmacist must also be involved in the collection of data that might be useful in longitudinal pharmacoepidemiological studies. There is a trendy rule where manufacturers deem their drugs as safe until proven harmful, and the regulatory authorities consider every drug might be harmful 
until proven safe. As a matter of fact, the pharmacists should consider both. Their role is not just confined to reporting adverse events but there has to be a proactive approach in preventing the drug related adverse events.

\section{STAYING VIGILANT}

One of the safety concerns is timely identification of the early warnings of side effects. The process is usually dependent on doctors suspecting something and trying to find an association between the drug and disease. This gives an opportunity for the pharmacists to step in and make a difference by identifying the initial users of new drugs through prescriptions and to monitor systematically rather than waiting for someone to recognize a possible adverse effect. This concept is better known as prescription event monitoring.

In addition to the pharmacist's responsibilities relating to the reporting of adverse events, they can also involve themselves in areas such as record keeping, education and monitoring the over-the-counter drugs. A major step in empowering the pharmacists is by providing access to the medication records of the patient, thereby maximizing the benefit and minimizing the risks of medication use. As a result, assessments of potential drug interactions and any adverse event are possible. In case of changes in the labeling or when a drug has been withdrawn from the market, it becomes absolutely necessary for the pharmacist to ensure that patients get a change in therapy but continue to take medications for chronic conditions.

\section{IN CLINICAL PRACTICE}

Pharmacists are one of the major stakeholders along with the physicians, patients and other healthcare professionals. Pharmacists play a key role in management and prevention of the adverse events associated with the drug. Safety concerns are often considered to be implied when drugs are approved or authorized. The scope has widened now. It includes all safety-related activity right from the moment humans are first exposed to the new drug. It should be noted that the pharmacists has an added advantage of getting in direct contact with the patients who may not be involved in the clinical trials for ethical reasons.

To be more precise, hospital pharmacists can play a significant role in ADR reporting because the most serious adverse drug events occur in hospitals. In addition, a number of publications reiterate the fact that adverse events account for a considerable percentage of all hospital admissions. Pharmacists can actually help in substantially reducing the incidence of the adverse events by recording as well as timely communication of adverse events occurring in hospitals to further control the harmful effects of these events. This can be achieved through direct involvement in patient care and a proper reporting system in place. The adverse event information obtained from hospitals can be quite advantageous because of their high-quality documentation. In fact, hospital pharmacists have access to sophisticated computer systems and databases which augers well for effective retrieval of information.

\section{INDIAN FRAME WORK}

The Central Drugs Standard Control Organization (CDSCO), Directorate General of Health Services under the aegis of the Ministry of Health and Family Welfare, Government of India in collaboration with Indian Pharmacopeia Commission (IPC), Ghaziabad has initiated a nation-wide pharmacovigilance programme as Pharmacovigilance Programme of India (PvPI) in 2010. The programme is being coordinated by IPC as a National Coordinating Centre (NCC). The centre operates under the supervision of a Steering Committee which has the Drug Controller General of India (DCGI) as its ex-officio chairman and the Officer-in-Charge (New Drugs), CDSCO, New Delhi as its ex-officio Member Secretary. PvPI currently has 150 functional ADR monitoring centers (AMCs) across the country. With increased nutritive interest shown by Government, regulators, and industry, the numbers are expected to soon increase involving private and government medical colleges as well as other hospitals.

\section{ACCESSIBILITY}

It can be observed that under reporting can be significantly reduced by actively involving pharmacists in the surveillance of drug safety. It is highly recommended to increase the participation of pharmacists especially in a country like ours, where the unawareness is accounted for the decline in reporting. Negligence of reporting is also considered to be a factor for the steady dearth in reporting and seen as a major setback among Indian HCPs. The challenge now remains in creating awareness.

Back in the old days, information about possible adverse effects of drugs was spread through medical literature which was then the available effective way. Accessibility is far more accessible now and the needed information just a swipe away. Information technology can be used constructively to improve communication. Pharmacists have quick access to a pool of information on medication safety. PvPI now has a dedicated website with a PvPI toolkit for the stakeholders. PvPI has launched toll 
free helpline (1800-180-3024) to facilitate reporting of adverse reactions. There are various national level workshops held to provide training to the stakeholders and generating awareness. Moreover, it has become almost imperative for pharmacists to use the Suspected Adverse Drug Reaction Reporting (SADRR) Forms to report suspected adverse drug events. The interaction of much of the stakeholders with the AMCs is primarily through the SADRR forms. This will be helpful for the AMCs to maintain a database of all the adverse events associated with the drug. With reference to the recent Drugs Technical Advisory Board (DTAB) recommendation, pharmacists or trained medical officers are also entrusted with the responsibility of maintaining a dedicated pharmacovigilance cell in all pharmaceutical companies. Suggestions are being proposed at various levels to consider incorporation of PV concepts in education curriculum.

\section{TAKE A LEAP}

Aspirants who wish to take up pharmacovigilance as their career; this might be the right time as future can see a demand in qualified pharmacovigilance specialists. Recently drug safety has earned concerns by regulatory authorities worldwide. Stringent laws are being adopted. Global pharmaceutical companies are looking at India for their pharmacovigilance activities. This can be an added benefit to the existing outsourcing hub. There are various openings among leading PV service providers, clinical research organizations, and IT companies. India is one of the largest pharmaceutical industries with many major global players. Pharmaceutical companies are now setting up in-house units and they are in need of experts. With more AMCs being added, medical colleges and hospitals may see an ardent need for trained professionals. Besides, there are also chances to work for regulatory authorities like DCGI/CDSCO. The industry is all set to see much growth both in private as well as Government sector.

\section{OBSERVATION}

A life saving drug today can be banned tomorrow. It is very unlikely that harm of medicines can always be predicted and prevented but limiting the numbers is surely approachable. A pharmacist can perhaps be entrusted as an effective tool in collection and reporting of ADRs for they are present at all levels of medical care, right from community pharmacy to primary healthcare centers, government hospitals to corporate hospitals. Most importantly, pharmacies work as a department at affordable and approachable locations. This can serve as best takeaway point as pharmacists are actively involved in the final stages of patient care. Pharmacists can create a trusted environment by counseling patients to reduce medication errors, improve safety and quality of care; above all, it gets all the attention a pharmacist deserves.

\section{EPILOGUE}

Getting the pharmacists recognized as providers would help overcome the barrier to incorporate their role in the healthcare team. At the same time, pharmacists should step out; challenge themselves to take up the responsibilities while working in complex healthcare setting. Stay tuned, re-invent yourselves and be prepared. A budding pharmacist should realize clinical pharmacy is not just drug-drug, drug-food interaction, but it is also tracking adverse drug effects, reducing medication errors, monitoring patients' compliance, counseling patients. Healthcare is all about collective accomplishment and pharmacist do deserve a little credit. A bit of us lie in contributing back to society and if that giving involves our profession, what better a service can it be!

\section{CONFLICT OF INTEREST}

The authors declare no conflict of interest.

\section{Highlights of Paper}

- Pharmacists form the core of the interdisciplinary healthcare system where safety monitoring is an integral part of clinical practice.

- Monitoring the ongoing safety of medicines is part of their professional practice.

- This will be a proactive approach in preventing drug-related adverse events.

- It offers an added advantage of in-person contact between the pharmacist and patients.

- Underreporting can be significantly reduced with active participation of pharmacists.

\section{Author Profile}

- Dr J Vijay Venkatraman is a Diabetologist, Drug Safety Physician and Entrepreneur. He holds a MBA degree in Services Management and has been awarded the Fellowship of the Pharmaceutical Information \& Pharmacovigilance Association, United Kingdom. He is a Consultant Diabetologist at Diab-at-ease Clinik, KTVR Group Hospital, Coimbatore.

- Dr Vijay founded Oviya MedSafe, a global Pharmacovigilance Consulting \& Drug Safety Services company incorporated in India in 2012 and London in 2014. Dr Vijay is an invited speaker in national and international conferences on Drug Safety as well as on Diabetology. He has published in many national \& international journals and magazines. 


\section{REFERENCES}

1. Waller, Patrick. An Introduction to Pharmacovigilance. 1st Ed. UK. WileyBlackwell; 2010.

2. Van Grootheest AC, de Jong-van den Berg LTW. The Role of Hospital and Community Pharmacists in Pharmacovigilance. Research in Social and Administrative Pharmacy 2005. 126-33.

3. Roohi Kesharwani, Devendra Singh, Vishal Jacob. Pharmacovigilance: The Emerging Trend and its Future Prospects. The Global Journal of Pharmaceutical Research 2013; 2(1): 1561-84.

4. Kumar A. Past, present and future of pharmacovigilance in India. Systematic Reviews in Pharmacy 2011; 2(1): 55-8.
5. Rama. P, Prudence Rodrigues A, Archana Georgy. Pharmacovigilance: Perspectives and future challenges in Indian scenario. Asian Journal of Pharmaceutical and Clinical Re-search 2011; 4(4):1-4.

6. Janaki Torvi R, Rajesh Hunashal. Pharmacovigilance. International Journal of Pharma and Bio Sciences 2011; 2(4): 95-101.

7. Ankur Rohilla, Nishant Singh, Vipin Kumar, Mohit Kumar Sharma, Amar jeet Dahiya, Ashok Kushnoor. Pharmacovigilance: Needs and Objectives. Journal of Advanced Pharmacy Education \& Research 2012; 2(4): 201-4. 